\title{
Abdominal Enhanced Computed Tomography Image by Artificial Intelligence Algorithm in the Diagnosis of Abdominal Aortic Aneurysm
}

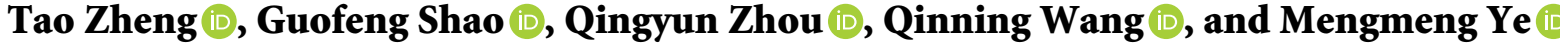 \\ Department of Cardiothoracic and Vascular Surgery, Ningbo Medical Center Lihuili Hospital, Ningbo 315000, Zhejiang, China \\ Correspondence should be addressed to Guofeng Shao; 1651010038@xzyz.edu.cn
}

Received 6 October 2021; Revised 12 November 2021; Accepted 1 December 2021; Published 28 December 2021

Academic Editor: M Pallikonda Rajasekaran

Copyright (c) 2021 Tao Zheng et al. This is an open access article distributed under the Creative Commons Attribution License, which permits unrestricted use, distribution, and reproduction in any medium, provided the original work is properly cited.

\begin{abstract}
The purpose of this study was to investigate the clinical value of CT angiography (CTA) images processed by the segmentation denoising technique based on deep convolution neural network algorithm in the diagnosis of abdominal aortic aneurysm (AAA) and the detection of disease changes. A total of 98 patients with ruptured AAA were retrospectively selected as the study subjects. Patients were grouped according to whether the CTA images were optimized, the images receiving artificial intelligence segmentation and denoising were set as the observation group, and the CTA images without optimization were set as the control group. The detection and diagnosis effects of CTA images before and after the treatment were compared. The surgical results were used as the standard to analyze the diagnostic effect, and the maximum diameter measurement results of AAA and the proportion results of intraluminal thrombus (ILT) were compared. Although the sensitivity and accuracy of diagnosis in the observation group (97.73\% and 94.9\%) were higher than those in the control group (95.45\% and 92.86\%), there was no significant statistical significance $(P>0.05)$. When the diameter of AAA was no less than $5 \mathrm{~cm}$, all results showed that the coverage percentage of intraluminal thrombus (ILT) was over 50\%. When the diameter of AAA was less than $5 \mathrm{~cm}$, only $55.56 \%$ of the results showed that the percentage of ILT coverage was over $50 \%$, with considerable differences $(P>0.05)$. According to the results of the study, it was found that there was a certain relationship between the thrombus coverage of the abdominal aortic wall and the growth rate of AAA. The deep convolution neural network algorithm had a certain effect on the treatment of CTA, but it is not obvious. However, CTA had a better clinical diagnostic effect on AAA.
\end{abstract}

\section{Introduction}

Abdominal aortic aneurysm (AAA) is a common aneurysm. It is mainly caused by the external dilation of the damaged arterial wall caused by the impulse of blood flow $[1,2]$. The disease is very common in the elderly, among which the incidence ratio between male and female is $4: 1-6: 1$, and the incidence will gradually increase after the age of 50 [3]. According to clinical statistics, the incidence of AAA in the general population is relatively low. In elderly people suffering from other atherosclerotic diseases and hypertension, AAA has a high incidence rate, but it occasionally occurs in young people [4-6]. As the disease progresses, about $80 \%$ of patients are at risk of dying as the tumor grows larger and ruptures. Even with surgical repair, the probability of death is up to $50 \%[7,8]$. Patients with AAA diameter no less than
$5 \mathrm{~cm}$ or accelerated increase in tumor volume within a short time should receive intervention treatment. Moreover, AAA patients with a diameter of $3-5 \mathrm{~cm}$ need regular vascular monitoring and risk assessment [9-11]. Currently, the main technique used to diagnose AAA is computed tomography angiography (CTA). CTA, which combines CT enhancement techniques with thin-section, wide-range, and rapid scanning techniques, clearly shows the vascular details of various parts of the body through reasonable postprocessing. It has the characteristics of noninvasive and simple operation and has important application value for the detection of vascular variation, vascular diseases, and the relationship between lesions and vessels. It has high spatial resolution and good image quality; however, due to the influence of medical imaging technology, contrast agent measurement, scanning time, and other factors, the image still has noise 
pollution, resulting in that the image details cannot be clearly displayed [12]. In addition, with the increase of clinical data, there will be certain errors and low reproducibility in disease assessment relying only on the experience of doctors [13].

However, with the rapid development of artificial intelligence (AI) technology such as machine learning, deep learning has made great breakthroughs in speech recognition, image recognition, and other fields and has been closely combined with the medical industry [14]. Especially, it occupies an important position in the field of medical imaging applications. Deep learning plays a crucial role in the segmentation of medical images [15]. Image segmentation is the foundation and key step of medical image analysis, 3D reconstruction, dose calculation, etc., and the segmentation effect directly affects the subsequent analysis and diagnosis [16]. Therefore, deep learning algorithms should have good performance. Studies found that convolutional neural network algorithm has a good application effect in segmentation and noise removal of medical images [17]. However, it was also proposed that conventional convolutional neural network algorithms would lead to reduced training efficiency when the number of training parameters was large and often ignore the low-level semantic information of the bottom layer, and the effect of segmentation or denoising will also be reduced [18, 19]. Therefore, a deep convolutional neural network algorithm was proposed, which adopts the way of local link and parameter sharing. Compared with the traditional neural network, the network parameters are greatly reduced and the depth of the network is maintained, so that the model has a good generalization ability [20].

In this study, the deep convolutional neural network algorithm was employed to segment and denoise the CTA images of AAA patients, which were then applied to the diagnosis and detection of disease progression in AAA patients, so as to evaluate the clinical application value of this technology in AAA. It was hoped to provide ideal monitoring means to reduce the mortality rate of AAA and improve the cure rate.

\section{Methods}

2.1. Study Subjects. In this study, 98 patients with ruptured AAA in the hospital were retrospectively selected from August 2019 to April 2021 as the study subjects, among which there were 80 males and 18 females, with an average age of $(66.3 \pm 14.7)$ years old. The deep convolutional neural network algorithm was adopted to segment and denoise the CTA images of all patients during the treatment. The processed images were set as the observation group, which were compared and analyzed with the inspection result of CTA image before processing (set as control group). This study had been approved by the ethics committee of the hospital.

Inclusion criteria were as follows: (a) all the selected patients were over 40 years old and below 80 years old; (b) the CTA images of the patients were kept intact; and (c) ultrasound or CT diagnosis showed that the diameter of the aorta in the lower renal abdomen was no less than $3 \mathrm{~cm}$.
Exclusion criteria were as follows: (a) patients with malignant tumors, other serious cardiovascular diseases, and infectious diseases were excluded; (b) patients who received treatment of oral anticoagulants or steroids were excluded; (c) patients with recent surgical history and acute thrombotic disease within three months were excluded.

2.2. CTA Image Optimization Model Based on Deep Convolutional Neural Network Algorithm. In this study, the deep convolutional neural network algorithm was utilized to establish the segmentation and denoising model, which was as follows.

First, the segmentation of CTA images was carried out.

JFCM algorithm was used to filter features in feature map screening. In this process, preclassification was screened to improve the accuracy of classification results. The standard selection is shown in the following formula:

$$
\frac{F\left(q_{\varphi o} \in W_{i j} \wedge \Omega_{\varphi o}=\Omega_{i j}\right.}{n \times n}>\partial,
$$

where $(i, j)$ is the position of feature graph and $\Omega_{i j}$ is the mark of pixel $p_{i j}$.

The convolution layer is set as $e$, and then the feature map of ultrasonic detection image was input in the $e-1$ layer, which is expressed as follows:

$$
R(t, u)=\sum_{b=1}^{B} X^{(t, u, b)} \otimes N^{(t-1, b)}+j^{(t, p)},
$$

where $X^{(h, p, b)}$ is the convolution kernel, $j^{(t, p)}$ is the bias, and $b$ is the number of feature maps.

To improve the expression ability of data characteristics in the process of network computing, some experts proposed nonlinear activation function. In this study, the relatively common and fast rate ReLu function was adopted as the activation function, which is expressed in formula (2), and its corresponding derivative function is expressed as follows:

$$
\begin{aligned}
f(x) & =\max (0, x) \\
f^{\prime}(x) & = \begin{cases}x, & x>0 \\
0, & x \leq 0\end{cases}
\end{aligned}
$$

It is supposed that $H$ is the ultrasound image of the central nervous system of the fetus, all pixels in the image $H$ are expressed by $T$, and the collection form is $\left(g_{1}, g_{2}, \ldots, g_{n}\right)$. The part of the image that needs to be identified and optimized is represented by $U$, expressed as $\left(h_{1}, h_{2}, \ldots, h_{n}\right)$, and then the probability of corresponding output for the pixel of the $j$-th channel is as follows:

$$
V\left(g_{n}=h_{j}\right)=\frac{1}{E} \exp \left[v\left(h_{j}\right)\right]
$$

where $v\left(h_{j}\right)$ is the value of $u_{j}, E$ is the regularization term, and the predicted value $y_{n}$ obtained by $s_{n}$ is expressed as $y_{n}=\arg \max \left[p\left(s_{n}=u_{j}\right)\right]$, whose corresponding loss function is expressed as follows: 


$$
L=-\frac{1}{c d} \sum_{n} \sum_{j} y_{n j} n\left[p\left(s_{n}=h_{j}\right)\right] l \text {. }
$$

Batch normalization (BN) is one of the methods of optimizing neural networks, which can reduce the difficulty of learning and can reduce the running time during model training. This method preprocesses the previous step in advance when processing each layer and standardizes the data, which was employed in this study to improve the algorithm based on deep convolutional neural network algorithm.

The expression of $\mathrm{BN}$ is as follows:

$$
\bar{L}^{i}=\frac{L^{(i)}-Z\left[L^{i}\right]}{\sqrt{\operatorname{Var}\left[L^{(i)}\right]}}
$$

where $Z\left[L^{i}\right]$ is the average value of each group of sample $\mathbb{S}$ and $\operatorname{Var}\left[L^{(i)}\right]$ is the variance, but there are still some problems in the expression of formula (5), so new parameters $\beta$ and $\mu$ are introduced into the above formula.

The expression of formula (6) of the new $\mathrm{BN}$ is as follows:

$$
\begin{aligned}
Q^{i} & =\beta^{(i)} \bar{L}^{(i)}+\mu^{(i)}, \\
\beta^{(i)} & =\sqrt{\operatorname{Var}\left[L^{(i)}\right]}, \\
\mu^{(i)} & =Z\left[L^{i}\right], \\
Z & =\frac{\beta}{\sqrt{\operatorname{Var}[L]+\varphi}} \cdot X+\left(\mu-\frac{\beta Z(L)}{\sqrt{\operatorname{Var}[L]+\varphi}}\right) .
\end{aligned}
$$

$\mathrm{BN}$ belongs to a single layer and is usually placed behind the base layer of the roll and in front of the activation function.

The segmentation process is shown in Figure 1.

Next, denoising processing was performed. It is supposed that the CTA image of the segmented AAA patient is $F=\left\{f_{v}\right\}_{v=1}^{v}$, the image without noise is $m_{v}$, and the image with noise is $n_{v}$.

$$
f_{v}=m_{v}+n_{v}
$$

An image block $P_{i, j}$ with a pixel center $j$ and an area of $m \times m$ is defined, and $z$ approximate blocks $\left\{p_{i, j, v}\right\}_{i=1}^{z}$ were found in the image $f_{v}$. A matrix $P_{i, j}$ of $n^{2} \times m$ as the following formula is defined by concatenating the column vectors of the approximate block $p_{i, j, v}$ and proposing $P_{i, j, v} \in R^{n^{2}}$.

$$
P_{j, v}=\left(P_{1, j, v}, P_{2, j, v}, \ldots, P_{z, j, v}\right) .
$$

Equation (10) is expressed as follows:

$$
P_{i, j}=M_{i, j}+N_{i, j}
$$

The adaptive median filter algorithm was used to preprocess the noisy data, and then the corresponding matrix of the noise-free image was obtained through the solved minimization problem, and finally the overlapping matrix was merged to obtain a noise-free MRI image.

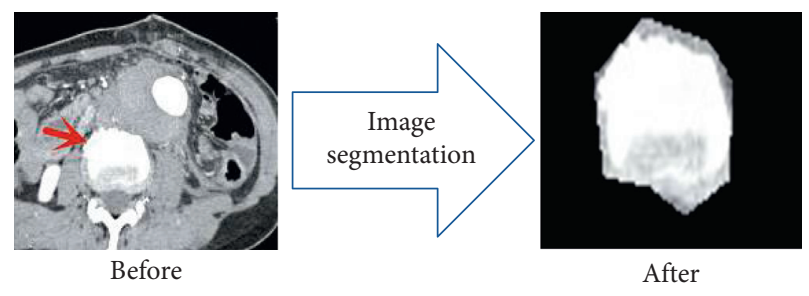

Figure 1: CTA image segmentation process (left: before segmentation; right: after segmentation).

The denoising process is shown in Figure 2.

This study was a retrospective analysis, so there was no need to conduct CTA examination again. It was only necessary to collect the CTA images from the computer system and then process them with segmentation and denoising technology. Finally, the images were reviewed to understand the development of the disease. According to the number of days from prehospital examination to tumor rupture, CTA images at three time points were selected for study and analysis, namely, before admission, the day before rupture, and the middle time (MT) from the day before admission to the day before rupture.

\subsection{Observation Indexes.}

(i) The results of surgical diagnosis were taken as the gold standard, and the accuracy of AAA diagnosis results of CTA images between the observation group and the control group was compared before hospitalization.

(ii) The maximum diameter of AAA in the two groups of images was measured two months before rupture, and the limit of $5 \mathrm{~cm}$ was set to compare the difference of measurement results between the two groups of images.

(iii) Intraluminal thrombus (ILT) was found in most AAA patients, and its morphology distribution was different. Many studies showed that ILT plays an important role in the progression of AAA. Therefore, ILT was observed in this study. During the study, AAA ILT was divided into four parts according to its coverage percentage: $0-25 \%, 25 \%-50 \%, 50 \%-75 \%$, and $75-100 \%$. The AAA diameter was divided into two groups with $5 \mathrm{~cm}$ as the limit: $<5 \mathrm{~cm}$ and $\geq 5 \mathrm{~cm}$ (requiring intervention), and the characteristics of ILT in the two groups were analyzed.

2.4. Statistical Methods. SPSS 22.0 was used for data entry, sorting, and statistical analysis. Counting data were compared by the $\chi^{2}$ test. The measurement data were compared by the $t$-test. Analysis of variance (ANOVA) was used for comparison of multiple sample means, the LSD method was used for homogeneity of variances, and the Dunnett T3 method was used for heterogeneity of variances. $P>0.05$ indicated statistically significant difference. 


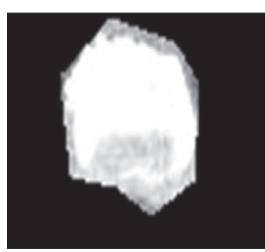

Before

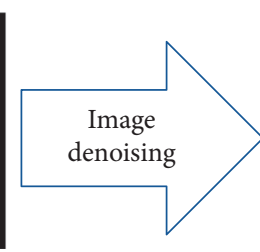

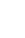

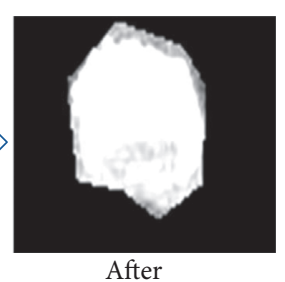

After
Figure 2: CTA image denoising process (left: before denoising; right: after denoising).

\section{Results}

3.1. General Data Statistics of Patients. The mean age of the 98 subjects in this study was 66.3 years, ranging in age from 48 to 78 years. There were 3 cases (3.06\%) between 40 and 49 years old, 16 cases (16.33\%) between 50 and 59 years old, 23 cases $(23.47 \%)$ between 60 and 69 years old, and 56 cases (57.14\%) between 70 and 79 years old. Through comparison, it was obvious that the proportion of patients aged between 70 and 79 was higher than other age groups, as shown in Figure 3.

Among the 98 subjects, 80 cases were males (81.63\%) and 18 cases were females $(18.37 \%)$, with a ratio of $4.44: 1$. The incidence rate of males was much higher than that of females after comparison, as shown in Figure 4.

\subsubsection{Disease Course and AAA Diameter Distribution.} Figure 5 shows the distribution of disease course of 98 patients, including 23 patients with disease course of 1-3 months, 40 patients with disease course of 4-6 months, 21 patients with disease course of 7-9 months, and 14 patients with disease course of 10-12 months. AAA diameter distribution: 72 patients with AAA diameter $\geq 5 \mathrm{~cm}$ and 26 patients with diameter $<5 \mathrm{~cm}$.

3.2. Prehospitalization Diagnosis. Table 1 shows the statistical results of CTA imaging and surgical diagnosis of the two groups before hospitalization. There were seven patients in the two groups of CTA imaging examination results. The sensitivity, specificity, and accuracy of the observation group were $97.73 \%, 70 \%$, and $94.9 \%$, respectively. The sensitivity, specificity, and accuracy of the control group were $95.45 \%$, $70 \%$, and $92.86 \%$, respectively. After comparison, there was no significant difference in the sensitivity, specificity, and accuracy of CTA images for AAA between the two groups $(P>0.05)$, as shown in Figure 6.

\subsection{Maximum Diameter Measurement Result Statistics.} Table 2 shows the diameter distribution of AAA lesions detected by CTA imaging in the two groups of AAA patients. After analysis, there were 27 patients (27.55\%) with AAA diameter $<5 \mathrm{~cm}$ in the observation group and 25 patients $(25.51 \%)$ with diameter $<5 \mathrm{~cm}$ in the control group, and there was no significant difference $(P>0.05)$. For patients with diameter no less than $5 \mathrm{~cm}$, there were 71 cases in the observation group $(72.45 \%)$ and 73 cases in the control

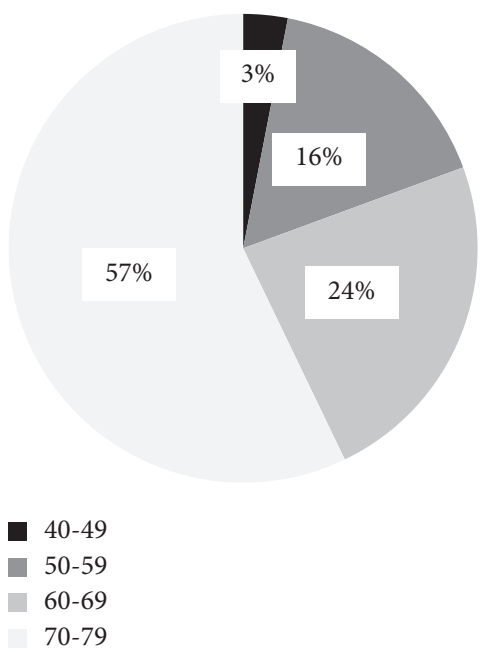

Figure 3: Distribution of age at onset.

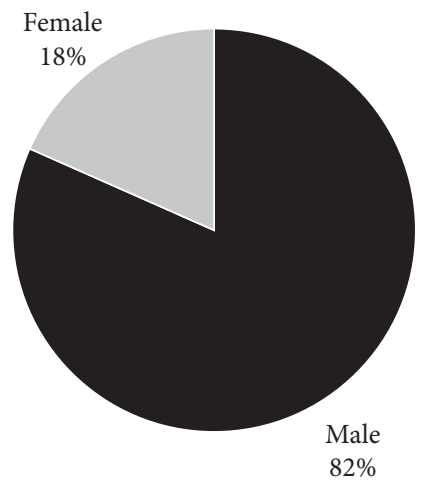

Figure 4: Male and female incidence ratios.

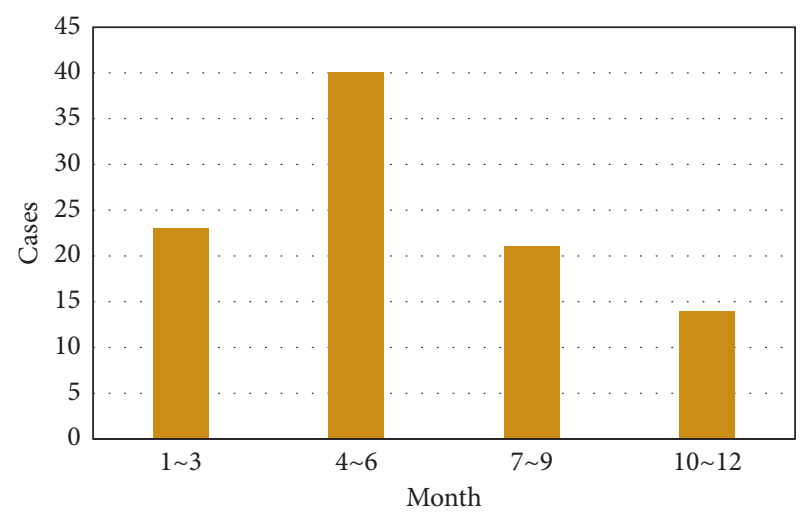

FIgURE 5: Distribution of patients' disease course.

group (74.49\%), with no significant difference $(P>0.05)$, as shown in Figure 7. It was suggested that the CTA images before and after treatment had basically the same effect in the detection of lesion diameter. However, there were significant differences in the proportion of distribution within the group $(P<0.05)$, as shown in Figure 8. This comparison showed that the tumor body diameter of most patients with ruptures reached $5 \mathrm{~cm}$ or above. 
TABLE 1: Statistics of diagnostic results before hospitalization.

\begin{tabular}{ccccc}
\hline \multirow{2}{*}{ Group } & & \multicolumn{2}{c}{ Surgery confirmed } & \multirow{2}{*}{ Total } \\
& & Positive & Negative & \\
\hline \multirow{2}{*}{ Observation group } & Positive & 86 & 3 & 89 \\
Total & Negative & 2 & 7 & 9 \\
& & 88 & 10 & 98 \\
Control group & Positive & 84 & 3 & 86 \\
Total & Negative & 4 & 7 & 12 \\
& & 88 & 10 & 98 \\
\hline
\end{tabular}

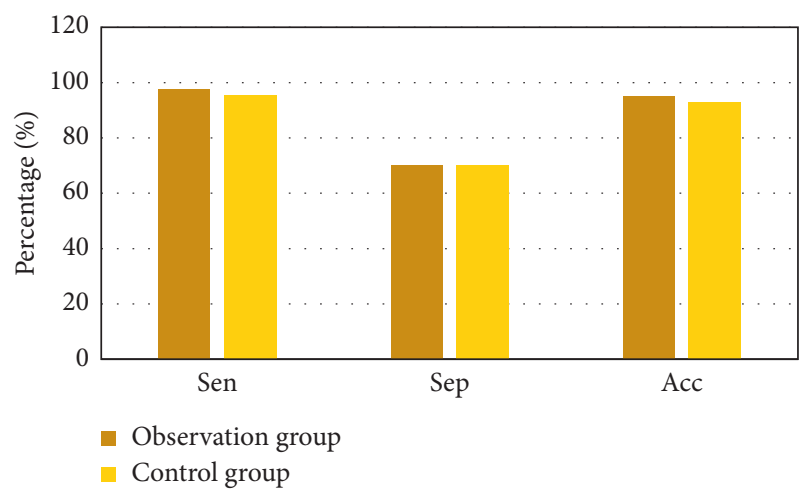

Figure 6: Comparison of diagnostic accuracy between the two groups of CTA images.

TABLE 2: Diameter measurement result statistics.

\begin{tabular}{ccccc}
\hline Group & & \multicolumn{2}{c}{$\begin{array}{c}\text { Observation } \\
\text { group }(n=98 \\
\text { cases })\end{array}$} & Total \\
& $<5 \mathrm{~cm}$ & $\geq 5 \mathrm{~cm}$ & \\
\hline Control group $(n=98$ cases $)$ & $<5 \mathrm{~cm}$ & 10 & 15 & 25 \\
Total & $\geq 5 \mathrm{~cm}$ & 17 & 56 & 73 \\
& & 27 & 71 & 98 \\
\hline
\end{tabular}

3.4. Distribution ofILT. It was found that 94 of the 98 patients had ILT (since the results of the two groups were consistent, no comparison was made here, and only the results of the observation group were used as an example for illustration), as shown in Figure 9. The CTA of ILT in all patients presented uniform attenuation, and the distribution characteristics of ILT are shown in Table 3. The distribution of ILT types was analyzed according to AAA diameter no less than $5 \mathrm{~cm}$ and less than $5 \mathrm{~cm}$. When AAA diameter was no less than $5 \mathrm{~cm}$, all results showed that ILT coverage percentage was over $50 \%$. When AAA diameter was less than $5 \mathrm{~cm}$, only $55.56 \%$ of the results showed that ILT coverage percentage was over $50 \%$, and the difference was substantial $(P<0.05)$.

\section{Discussion}

In this study, the detection and analysis of AAA were compared between CTA images and CTA images based on deep convolutional neural network algorithm. It was found that the sensitivity, specificity, and accuracy of CTA images

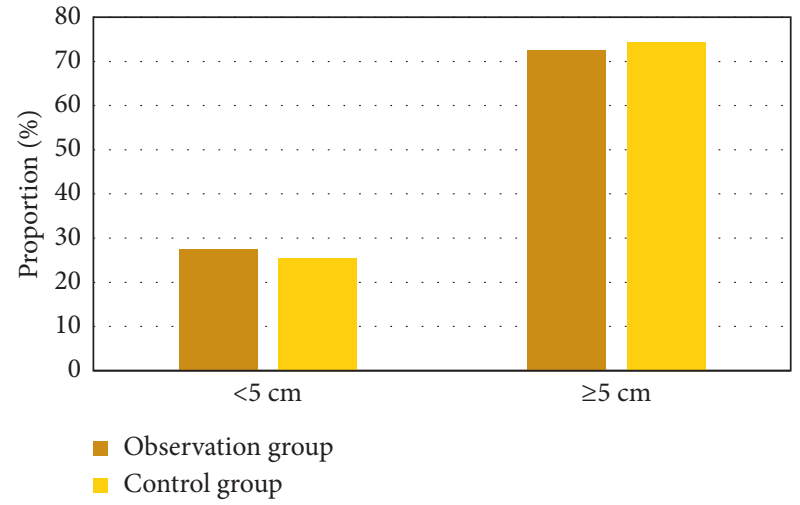

FIGURE 7: Comparison of distribution between groups.

before and after treatment for the diagnosis of AAA were $97.73 \% / 95.45 \%, 70 \% / 70 \%$, and $94.9 \% / 92.86 \%$, respectively, without significant differences. In addition, the accuracy of both CTA and CTA under deep convolutional neural network algorithm in the diagnosis of AAA was at a high level, which reflected that CTA had certain advantages in the diagnosis of AAA. Some experts discussed the clinical application value of 64-slice spiral CT angiography in the diagnosis and preoperative evaluation of AAA. The results showed that 64 -slice spiral CTA had the advantages of being noninvasive, rapid, and highly accurate and was one of the preferred imaging examination methods for AAA diagnosis and preoperative evaluation [22], which also reflected the good application value of CTA. Mohammad et al. (2019) [22] also used the convolutional neural network and Hough Circles algorithm to automatically segment, detect, and diagnose AAA. The results showed that the accuracy of the $\mathrm{CNN}$ aortic region detection classifier was $98.62 \%$, and Hough Circles algorithm could classify 120 aortic plaques according to diameter with 98.33\% accuracy. The Hough Circles algorithm can classify 120 aortic plaques based on their diameter, with an accuracy of $98.33 \%$. A study on deep learning of deep convolutional neural network algorithm to distinguish liver masses in dynamic contrast-enhanced CT showed that the median accuracy of differential diagnosis test data for liver masses was 0.84 . The median area under the operating characteristic curve of the receivers distinguishing categories $\mathrm{AB}$ and $\mathrm{CE}$ was 0.92 . It showed that deep convolutional neural network algorithm had high diagnostic performance in the differential diagnosis of liver masses under dynamic CT [23]. The above research showed the auxiliary advantages of deep learning algorithms in medical imaging, but this research failed to reflect them reasonably, which may be insufficient in the choice of algorithm performance. In addition, through the analysis of the general data of the patients in this study, it was found that the incidence of AAA in the age group of 50-79 years accounted for about $97 \%$, and the ratio of male to female was 4.44:1, indicating that the prone group of AAA was mainly the elderly, and the incidence of male is much higher than that of female. This is consistent with the epidemiological statistics of AAA diseases [24].

The occurrence and distribution of ILT was studied by using processed CTA images. Results showed that 94 of the 


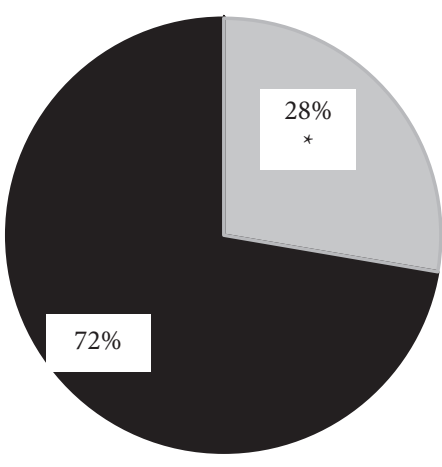

$<5 \mathrm{~cm}$

$\geq 5 \mathrm{~cm}$

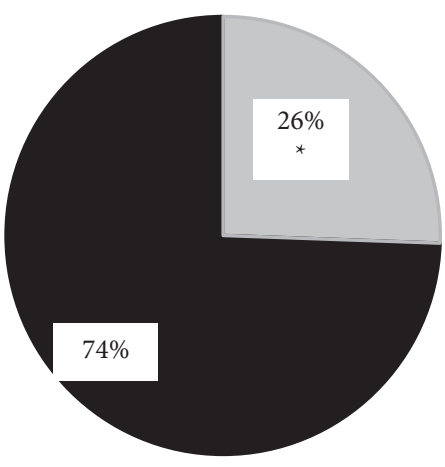

$<5 \mathrm{~cm}$

$\geq 5 \mathrm{~cm}$

(a)

(b)

Figure 8: Comparison of distribution within groups. (a) Observation group. (b) Control group. Note: “*” indicated that the difference was statistically significant $(P<0.05)$.

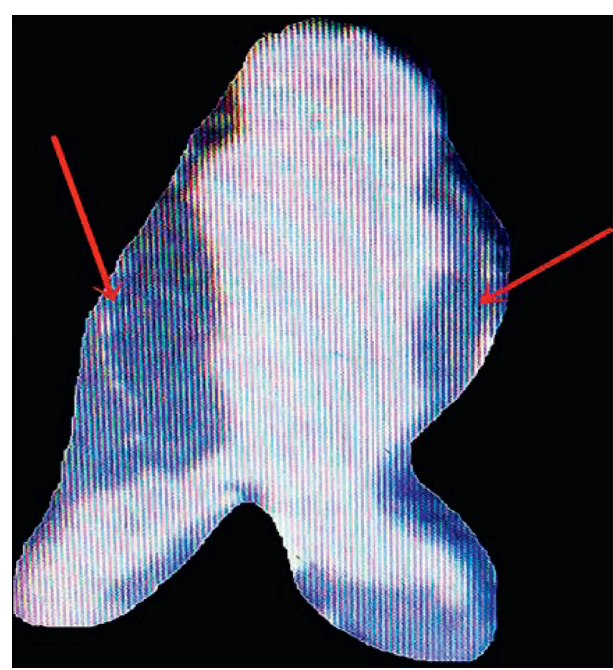

(a)

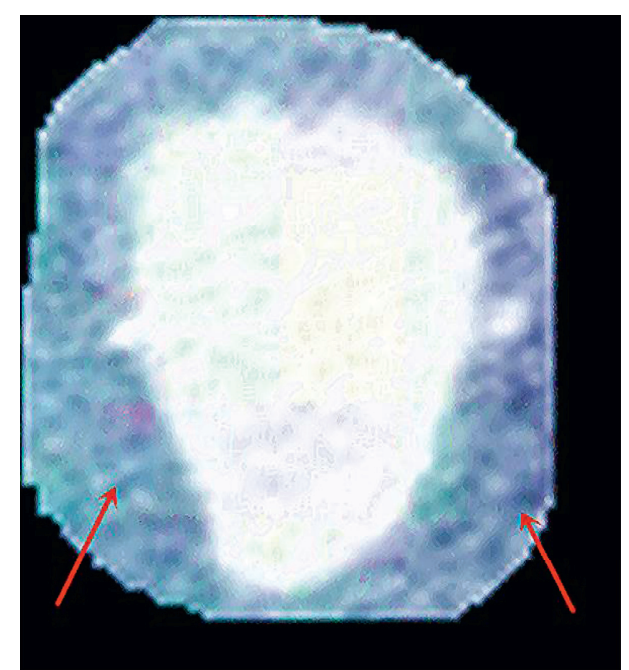

(b)

Figure 9: ILT in the abdominal aorta. (a) Coronal plane. (b) Cross section (red arrow is ILT).

TABLE 3: Distribution of ILT.

\begin{tabular}{lcc}
\hline Coverage & $<5 \mathrm{~cm}(n=2$ cases $)$ & $\geq 5 \mathrm{~cm}(n=71$ cases $)$ \\
\hline$>25 \%$ & $6(22.22)$ & $0(0.00)$ \\
$25-50 \%$ & $8(29.63)$ & $0(0.00)$ \\
Total & $14(51.83)$ & $0(0.00)$ \\
$50-75 \%$ & $8(29.63)$ & $25(35.21)$ \\
$<75 \%$ & $7(25.93)$ & $46(64.79)$ \\
Total & $13(55.56)$ & $71(100.00)$ \\
\hline
\end{tabular}

98 patients were with ILT, accounting for about $95.92 \%$, indicating that the incidence of ILT in AAA patients was very high. Relevant statistical results showed that most AAA patients had ILT in the abdominal aorta wall [25], which is consistent with the results of this study. The results also showed that the percentage of ILT coverage was over $50 \%$ when the AAA diameter was no less than $5 \mathrm{~cm}$. When the AAA diameter was less than $5 \mathrm{~cm}$, only $55.56 \%$ of the results showed that ILT coverage percentage was over $50 \%$. It was proposed that when the diameter of AAA was no less than $5 \mathrm{~cm}$, the rapid growth of AAA may be correlated with the degree of thrombus coverage in the abdominal aortic wall. This may be caused by hypoxia in the inflammatory state of AAA aortic wall. Studies revealed that high-density thrombosis in AAA detected by conventional CT was associated with AAA rupture [26, 27]. However, this study failed to compare the detection effect of CTA with conventional CT.

\section{Conclusion}

In this study, CTA images in the process of diagnosis and treatment of 98 patients in our hospital were used as the basis to compare the diagnostic effects of the images before and after process. According to the results of the study, there is a certain relationship between the thrombus coverage of 
the abdominal aortic wall and the growth rate of AAA. The deep convolution neural network algorithm has a certain effect on the treatment of CTA, but it is not obvious. However, CTA has a better clinical diagnostic effect on AAA. However, this study fails to reflect the advantages of artificial intelligence algorithm well, which is different from other research results, and it may be related to the unreasonable performance selection of the algorithm. There are also insufficient observational indicators in the study, which leads to imperfect results. In the future studies, we will improve it and hope to develop better detection technology to better diagnose and treat AAA patients. CTA examination has good accuracy in the diagnosis of AAA and is worthy of clinical application.

\section{Data Availability}

The data used to support the findings of this study are available from the corresponding author upon request.

\section{Conflicts of Interest}

The authors declare that they have no conflicts of interest.

\section{References}

[1] E. E. Joviliano, M. S. Ribeiro, and E. J. R. Tenorio, "MicroRNAs and current concepts on the pathogenesis of abdominal aortic aneurysm," Brazilian Journal of Cardiovascular Surgery, vol. 32, no. 3, pp. 215-224, 2017.

[2] H. Kuivaniemi, E. J. Ryer, J. R. Elmore, and G. Tromp, "Understanding the pathogenesis of abdominal aortic aneurysms," Expert Review of Cardiovascular Therapy, vol. 13, no. 9, pp. 975-987, 2015.

[3] B. W. Ullery, R. L. Hallett, and D. Fleischmann, "Epidemiology and contemporary management of abdominal aortic aneurysms," Abdominal Radiology, vol. 43, no. 5, pp. 1032-1043, 2018.

[4] R. Umebayashi, H. A. Uchida, and J. Wada, "Abdominal aortic aneurysm in aged population," Aging, vol. 10, no. 12, pp. 3650-3651, 2018.

[5] R. Umebayashi, H. A. Uchida, and J. Wada, "The aging population and research into treatments for abdominal aortic aneurysms," Acta Medica Okayama, vol. 73, no. 6, pp. 475-477, 2019.

[6] I. M. Nordon, R. J. Hinchliffe, I. M. Loftus, and M. M. Thompson, "Pathophysiology and epidemiology of abdominal aortic aneurysms," Nature Reviews Cardiology, vol. 8, no. 2, pp. 92-102, 2011.

[7] R. T. Schmitz, M. Keese, M. Hakimi et al., "Ruptured abdominal aortic aneurysm-epidemiology, predisposing factors, and biology," Langenbeck's Archives of Surgery, vol. 401, no. 3, pp. 275-288, 2016.

[8] F. R. Soares, O. N. Gomes, P. J. Oliveira et al., "Review on management and outcomes of ruptured abdominal aortic aneurysm in women," The Journal of Cardiovascular Surgery, vol. 59, no. 2, pp. 195-200, 2018.

[9] A. C. Hollingsworth, C. Dawkins, P. F. Wong, P. Walker, S. Milburn, and R. Mofidi, "Aneurysm morphology is a more significant predictor of survival than hardman's index in patients with ruptured or acutely symptomatic abdominal aortic aneurysms," Annals of Vascular Surgery, vol. 58, pp. 222-231, 2019.
[10] E. L. Verhoeven, M. R. Kapma, W. T. Bos et al., "Mortality of ruptured abdominal aortic aneurysm with selective use of endovascular repair," The Journal of Cardiovascular Surgery, vol. 50, no. 5, pp. 587-593, 2009.

[11] E. L. Leemans, T. P. Willems, C. H. Slump, M. J. van der Laan, and C. J. Zeebregts, "Additional value of biomechanical indices based on CTa for rupture risk assessment of abdominal aortic aneurysms," PLoS One, vol. 13, no. 8, Article ID e0202672, 2018.

[12] G. Carrafiello, C. Recaldini, D. Laganà, G. Piffaretti, and C. Fugazzola, "Endoleak detection and classification after endovascular treatment of abdominal aortic aneurysm: value of CEUS over CTA," Abdominal Imaging, vol. 33, no. 3, pp. 357-362, 2008.

[13] L. R. Philipp, D. J. McCracken, C. E. McCracken et al., "Comparison between CTA and digital subtraction angiography in the diagnosis of ruptured aneurysms," Neurosurgery, vol. 80, no. 5, pp. 769-777, 2017.

[14] H.-P. Chan, R. K. Samala, L. M. Hadjiiski, and C. Zhou, "Deep learning in medical image analysis," Advances in Experimental Medicine \& Biology, vol. 1213, pp. 3-21, 2020.

[15] M. H. Hesamian, W. Jia, X. He, and P. Kennedy, "Deep learning techniques for medical image segmentation: achievements and challenges," Journal of Digital Imaging, vol. 32, no. 4, pp. 582-596, 2019.

[16] Y. Chen, S. Hu, H. Mao, W. Deng, and X. Gao, “Application of the best evacuation model of deep learning in the design of public structures," Image and Vision Computing, vol. 102, Article ID 103975, 2020.

[17] S. K. Warfield, K. H. Zou, and W. M. Wells, "Simultaneous truth and performance level estimation (STAPLE): an algorithm for the validation of image segmentation," IEEE Transactions on Medical Imaging, vol. 23, no. 7, pp. 903-921, 2004.

[18] S. M. Anwar, M. Majid, A. Qayyum, M. Awais, M. Alnowami, and M. K. Khan, "Medical image analysis using convolutional neural networks: a review," Journal of Medical Systems, vol. 42, no. 11, p. 226, 2018.

[19] J.-H. Lee, D.-H. Kim, S.-N. Jeong, and S.-H. Choi, "Detection and diagnosis of dental caries using a deep learning-based convolutional neural network algorithm," Journal of Dentistry, vol. 77, pp. 106-111, 2018.

[20] J. Liu and H. Zhao, "Application of convolution neural network in medical image processing," Technology and Health Care, vol. 29, no. 2, pp. 407-417, 2021.

[21] J. Zhao, "Optimization of machine online translation system based on deep convolution neural network algorithm," Computational Intelligence and Neuroscience, vol. 2021, Article ID 7388825, 10 pages, 2021.

[22] J. S. Wilson, L. Virag, P. Di Achille, I. Karšaj, and J. D. Humphrey, "Biochemomechanics of intraluminal thrombus in abdominal aortic aneurysms," Journal of Biomechanical Engineering, vol. 135, no. 2, Article ID 021011, 2013.

[23] R. P. J. Budde, F. Huo, M. J. M. Cramer et al., "Simultaneous aortic and coronary assessment in abdominal aortic aneurysm patients by thoraco-abdominal 64-detector-row CT angiography: estimate of the impact on preoperative management: a pilot study," European Journal of Vascular and Endovascular Surgery, vol. 40, no. 2, pp. 196-201, 2010.

[24] Y. Qiu, Y. Wang, Y. Fan et al., "Role of intraluminal thrombus in abdominal aortic aneurysm ruptures: a hemodynamic point of view," Medical Physics, vol. 46, no. 9, pp. 4263-4275, 2019. 
[25] C. Zhu, J. R. Leach, Y. Wang, W. Gasper, D. Saloner, and M. D. Hope, "Intraluminal thrombus predicts rapid growth of abdominal aortic aneurysms," Radiology, vol. 294, no. 3, pp. 707-713, 2020

[26] R. Balm, R. Kaatee, J. D. Blankensteijn, W. P. T. M. Mali, and B. C. Eikelboom, "CT-angiography of abdominal aortic aneurysms after transfemoral endovascular aneurysm management," European Journal of Vascular and Endovascular Surgery, vol. 12, no. 2, pp. 182-188, 1996.

[27] M. R. Bashir, H. Ferral, C. Jacobs, W. McCarthy, and M. Goldin, "Endoleaks after endovascular abdominal aortic aneurysm repair: management strategies according to CT findings," American Journal of Roentgenology, vol. 192, no. 4, pp. W178-W186, 2009. 